Gerd Langguth beschreibt Horst Köhler als den „scheuen Patrioten“ (S. 275) - eine brillant gewählte Kurzformel für den neunten Bundespräsidenten. Zu keinem Zeitpunkt strebte Köhler dieses Amt an, der Anruf der Bundeskanzlerin am 3. April 2004, man habe sich auf ihn als Kandidaten verständigt, kam völlig unerwartet. Seine Distanz zum politischen Spiel der Kräfte blieb; ein Patriot war er aus der ganzen Tiefe seines Herzens. Seine Pflichterfüllung ließ keinen Zweifel gelten, dennoch blieb er der ökonomische Experte, dem man die Tür in die politische Arena geöffnet hatte, mithin „ein Seitenhineingenommener“ (S. 281).

Abschließend spannt Hans-Peter Schwarz den weiten geschichtlichen Bogen, der die Bundespräsidenten von Theodor Heuss bis Horst Köhler umfasst. Schwarz sieht einen historisch gewachsenen Widerspruch zwischen einer pluralistisch-zerfaserten Gesellschaft und dem Verlangen nach monarchistischer Grandezza und autoritärer Staatsgeschäftsführung. Die Bilanz fällt insgesamt negativ aus. Bedeutung und Einfluss des Bundespräsidenten „sind stark rückläufig“; sein Kapital „ist weitgehend verbraucht“ (S. 306). Für die Zukunft rechnet Schwarz mit einem Bedeutungsverlust des Amtes, „da das Amtsprestige des Bundespräsidenten durch Präsentation von Außenseitern oder Außenseiterinnen und Verlegenheitskandidaturen zwangsläufig abgewertet wird“ (S. 306).

Die Ereignisse des Jahres 2012 tauchen diese Worte in ein neues Licht. Am 18. März wurde Joachim Gauck zum Bundespräsidenten gewählt. Sein Amtsantritt wirkte wie eine Frischluftzufuhr und politischer Sekundenkleber zugleich. Durch seinen Verstand und seine Lebensklugheit, mithin durch die erhabene Autorität seiner Biographie gewinnt das Amt an Bedeutung. Hier liegt viel Stoff vor den Herausgebern, der nun in das vollständige Bild des Staatsoberhauptes einzuweben ist. Eine rasche Aktualisierung des Sammelbandes - mit analytisch geschärfter Perspektive - wäre sehr wünschenswert.

Helge F. Jani

\title{
Parteien in aller Vielfalt - drei empfehlenswerte Bände ohne Redundanzen
}

Holtmann, Everhard: Der Parteienstaat in Deutschland. Erklärungen, Entwicklungen, Erscheinungsbilder, Bundeszentrale für politische Bildung, Bonn 2012, 302 Seiten, € 4,50.

Korte, Karl-Rudolf und Jan Treibel (Hrsg.): Wie entscheiden Parteien? Prozesse innerparteilicher Willensbildung in Deutschland (ZPol-Sonderband 2012), Nomos Verlagsgesellschaft, BadenBaden 2012, 289 Seiten, $€ 34,-$.

Niedermayer, Oskar (Hrsg.): Handbuch Parteienforschung, VS Verlag für Sozialwissenschaften, Wiesbaden 2013, 926 Seiten, € 69,99.

Es ist allgemeiner Konsens, dass Parteien ein unverzichtbares Element westlicher Demokratien sind. Mit Artikel 21 des Grundgesetzes sind sie in der Bundesrepublik Deutschland entsprechend prominent verankert. In der Abwandlung eines Gemeinplatzes kann gesagt werden, dass ohne Parteien kein Staat zu machen ist. Drei 2012 und 2013 erschienene Bände wenden sich der analytischen Ist-Stands-Beschreibung des Parteienstaats in Deutsch- 
land, der innerparteilichen Demokratie und der Parteienforschung im Allgemeinen zu und bereichern damit den ohnehin sehr breiten Fachdiskurs.

Everhard Holtmann legt mit seiner Monographie ein Standardwerk der deutschen Parteiendemokratie nach der ersten Dekade des 21. Jahrhunderts vor. Er knüpft damit an die richtungsweisenden Arbeiten von Gerhard Leibholz, Wilhelm Hennis, Klaus von Beyme, Franz Walter und anderen an. ${ }^{1}$ Die diesen Werken zum Teil eigene Kritik am Parteienstaat und der Parteienpolitik greift der Autor in der zweiten Hälfte des Bandes auf. Dabei ist seine Darstellung eine Deskription der bisherigen Entwicklung und Analyse der gegenwärtigen Situation.

Holtmann folgt in seinen Ausführung den zentralen theoretischen Annahmen, dass es „feste Strukturen im Parteiensystem“ gibt, die von auf „sie bezogenen Kulturmustern“ getragen werden (S. 21). Im Bereich der Handlungsdimension geht es um das Verhältnis von Institutionen und Akteuren. Diese Faktoren sind zusätzlich einem ständigen Wechselspiel in der Zeit ausgesetzt (S. 22). Hieraus leitet der Autor seine bereits im Untertitel angedeutete Gliederung in Erklärungen, Entwicklungen und Erscheinungsbilder ab. Ziel ist es, zu zeigen, dass die Entwicklung in Deutschland „seit dem historischen Gründungsakt von 1949 in besonderer Weise pfadabhängig verläuft“ (S. 22). Dabei stellt er in Übereinstimmung mit der Fachmeinung fest, dass es zahlreiche „historische Überhänge“ der gegenwärtigen Parteiendemokratie gibt (S. 30).

Im Kapitel „Erklärungen“ widmet sich Holtmann theoretischen und definitorischen Fragen der Parteienforschung. Die Entstehung der Parteien und ihre Wechselwirkung mit der Gesellschaft werden vor dem Hintergrund der Theorie der historischen Pfadabhängigkeit nach Paul Pierson erläutert (S. 27 - 37). Die gängigen Begriffe und Konzepte von sozialem Wandel, „frozen party system“, Parteienfeindlichkeit und Probleme demokratischer Elitenherrschaft - um nur einige zu nennen - werden skizziert. Der zweite Teil „Entwicklungen“ geht auf die Geschichte der deutschen Parteien auf der Basis einer Typenbildung nach Max Weber, Otto Kirchheimer, Sigmund Neumann sowie Richard Katz und Peter Mair ein, um das Forschungsfeld übersichtlicher zu machen - allein 82 relevante Parteien seit 1980 (S. 61). Daran schließt sich eine Beschreibung der fünf Entwicklungsphasen des deutschen Parteiensystems und seiner Akteure an (S. $74-130)$.

Der dritte Abschnitt „Erscheinungsbilder“ macht wie oben angedeutet knapp die Hälfte der Ausführungen aus. Gerade in diesem Teil liegt das Potential für die weiterführende Forschung. In dichter Folge werden Fakten, Probleme und eben Erscheinungen des Parteienstaats in Deutschland erläutert. Der Rekurs auf die Theorie und das historische Erbe, aus denen sich selbst die „Parteienfeindschaft“ und in einer weniger martialischen Formulierung die „Parteienverdrossenheit“ (S. 147 - 149) erklären lässt, ist allgegenwärtig. Am Ende bleibt die Frage: Wie geht es weiter? Everhard Holtmanns Antwort ist im Nachgang der globalen Wirtschafts- und Finanzkrise: „Diese kritische Wegmarke hat die Politik (...) deshalb überzeugend gemeistert, weil sie konsequent auf dem Pfad weitergegangen ist, der marktwirt-

1 Vgl. Klaus von Beyme, Die politische Klasse im Parteienstaat, Frankfurt am Main 1993; Wilhelm Hennis, Auf dem Weg in den Parteienstaat: Aufsätze aus vier Jahrzehnten, Stuttgart 1998; Gerhard Leibholz, Strukturprobleme der modernen Demokratie, Karlsruhe 1958; Franz Walter, Vom Milieu zum Parteienstaat: Lebenswelten, Leitfiguren und Politik im historischen Wandel, Wiesbaden 2010. 
schaftliche Unternehmenspolitik, sozialpartnerschaftliche Regulierung und wohlfahrtsstaatliche Garantien in historisch bewährter Form zusammenführt." (S. 257) Konsequenz und Glaubwürdigkeit sind die beste Medizin gegen anhaltende Kritik.

Stilistisch ist das Werk gleichermaßen Auftragswerk für die politische Bildung und Beitrag zum Fachdiskurs. Es ist sehr verständlich geschrieben und arbeitet mit einem gebotenen $\mathrm{Ma}$ an Wiederholungen und Ergebnissicherung am Ende jedes Abschnitts oder Gedankengangs. Die Belegarbeit ist umfangreich und spiegelt den aktuellen - vor allem den deutschsprachigen - Forschungsstand wider und bezieht die Primärquellen wie Grundgesetz und Bundesverfassungsgerichtsurteile mit ein.

Der von Karl-Rudolf Korte und Jan Treibel herausgegebene Sonderband 2012 der Zeitschrift für Politikwissenschaft stellt die Frage der innerparteilichen Willensbildung in den Mittelpunkt der insgesamt zehn Beiträge von dreizehn Autoren. In die Auswahl der Untersuchungsobjekte wurde neben den langfristig etablierten Parteien - Bündnis 90/Die Grünen (Niko Switek), CDU (Oliver D'Antonio und Christian Werwath), CSU (Michael Weigl), Die Linke (Torsten Oppeland und Hendrik Träger), FDP (Jan Treibel) und SPD (Timo Grunden) - die Piratenpartei (Christoph Bieber und Markus Lewitzki) mit einbezogen. ${ }^{2}$

Dem Format des „Sonderbandes“ eines Periodikums scheinen einige erwähnenswerte Besonderheiten geschuldet. Ein Vorwort, Editorial oder Einleitungsartikel im klassischen Sinne fehlt. Dafür werden im ersten Beitrag sowohl der Forschungsstand als auch theoretische Zugänge in das Thema präsentiert. Der Hauptuntersuchungsgegenstand sind die innerparteilichen Machtstrukturen (Jan Treibel, S. 9). In der Tradition von Robert Michels, Max Weber sowie der Reflexion der bisherigen Forschung wird konstatiert, dass die Differenzierung nach Entscheidungsorten und -inhalten drei Ebenen in einer Pyramide zeichnet: Parteiführung, mittlere Parteielite und Parteibasis, in der die meisten Prozesse top-down verlaufen (Jan Treibel, S. 21). Alle Artikel zu den Parteien haben dann im Rahmen des skizzierten Forschungsstandes und Vorgehens einen eigenen Weg der Darstellung gewählt. Dies lässt sich ohne weiteres mit der Unterschiedlichkeit der Willensbildung - die sich zum Teil historisch unterschiedlich entwickelt hat - rechtfertigen (in der CSU beispielsweise der Abschied von der „Ein-Mann-Demokratie“ oder bei der Linken „Die Macht der Strömungen"). Damit entstehen eigenständige Porträts, die unterschiedlichen Untersuchungssettings folgen. So wird etwa im Falle der CDU Wert darauf gelegt, die Machtzentren zu identifizieren und ihnen zeit- und kontextspezifisch Ressourcen zuzuschreiben, während bei den Piraten - der kurzen Geschichte der Partei geschuldet - nur zwei „Fallskizzen“ im Mittelpunkt stehen. In einem Resümee der Porträts ist festzustellen, dass die innerparteilichen Entscheidungswege nicht immer dem top-down-Modell folgen. Gerade die jüngeren Parteien sind hier flexibler und haben, wie das Beispiel der Grünen zeigt, zum Teil bottom-up-Elemente fest verankert (Niko Switek, S. 148). Auf Grundlage der Daten der Deutschen Parteimitgliederstudie 2009 wird dies empirisch bestätigt. Der Wunsch der Parteibasis nach mehr Mitbestimmungsmöglichkeiten findet im Zuge der Adaptierung der Art der Piratenpartei bereits verschiedentlich Eingang in die Willensbildungsprozesse der etablierten Parteien und führt möglicherweise zukünftig zu einer stärkeren Beteiligung des unteren Teils der Pyramide

2 Der Autorenkreis hat aktuell oder perspektivisch bereits einige Publikationen zu den behandelten Parteien verfasst. Die meisten schreiben in der von Karl-Rudolf Korte bei der Nomos Verlagsgesellschaft herausgegebenen Reihe „Die Parteien der Bundesrepublik Deutschland“ zur selben Partei. 
(Ulrich von Alemann und Annika Laux, S. 263). Eine Form der Zusammenführung der Porträts findet erst in dem abschließenden Beitrag von Karl-Rudolf Korte „So entscheiden Parteien: Umfeld-Bedingungen innerparteilicher Partizipation“ statt. „Die Mitgliederparteien haben sich noch nicht aufgegeben. Durch Partizipationsanreize wollen sie den Mehrwert einer Parteimitgliedschaft erhöhen.“ Dabei bleibt jedoch abzuwarten ob diese „Rückkehr des Plebiszitären tatsächlich zu einer neuen Beteiligungsarchitektur bei den etablierten Parteien führt" (S. 281 f.).

Oskar Niedermayer legt als Herausgeber mit dem „Handbuch Parteienforschung“ ein Standardwerk der Zunft vor. Unter den Zwischentiteln: I. Grundlagen, II. Die Binnenansicht von Parteien, III. Parteien und ihre Umwelt, IV. Parteienanalyse, V. Parteiensystemanalyse, VI. Internationale Analysen (sowie einem Anhang) sind 31 Autoren mit 32 Beiträgen versammelt. Niedermayer zeichnet dabei mittel- oder unmittelbar für zehn Artikel, nach Seitenzahl für rund 25 Prozent, des Bandes verantwortlich. Der Autorenkreis rekrutiert sich größtenteils aus dem „Arbeitskreis Parteienforschung“ der Deutschen Vereinigung für Politische Wissenschaft (DVPW).

Die Themenblöcke des Bandes sind nachvollziehbar geordnet - von der Theorie zur Anwendung/Praxis - und ähnlich strukturiert. Grundsätzlich drängt sich der Vergleich mit dem „Handbook of Party Politics“3 auf, das erstmals 2006 erschienen ist.

Die Beiträge bieten einen Zugang zu allen wesentlichen Aspekten der Parteienforschung auf neuestem Stand. Trotz des natürlichen Blicks auf die Bundesrepublik sind alle Artikel außer die der Abschnitte IV. und V. ebenfalls als Basis für den internationalen Vergleich geeignet. Die Parteienporträts des Abschnitts IV. gehen nicht deckungsgleich vor, schauen aber gleichermaßen auf Geschichte, Mitglieder, Programmatik, Willensbildung und die Arbeit in Parlament respektive Regierung. Der im oben erwähnten „Handbook“ vorkommende Unterpunkt „Parties in the Future“ wird zum Teil in Abschnitt VI. integriert; der dort behandelte Aspekt „Parties in the media age“ wird als Bestandteil des Kontextes bei jeder Darstellung mitgedacht. „Cyber parties“ ist ein in Deutschland trotz der Erfolge der Piratenpartei noch nicht breit diskutierter möglicher neuer Idealtyp einer Partei.

Insgesamt sind alle Bücher, trotz unterschiedlicher Zielgruppen, für den Lehrbetrieb an der Universität zu empfehlen. Der Sonderband der ZPol 2012 ist aufgrund seiner sehr detaillierten Forschungsfrage und seiner Konzeption gewissermaßen ein Ergänzungsband zum Handbuch Parteienforschung. Everhard Holtmann schreibt für ein breites Publikum und leistet „nebenbei“ einen wichtigen Beitrag zur Debatte um die Rolle und das Bild von Parteien im 21. Jahrhundert. Die Tatsache, dass innerhalb kürzester Zeit drei Bücher zu einem bisher bereits gut durchdrungenen Gegenstand ohne nennenswerte Redundanz erschienen sind, zeigt die Vielfalt, die die Parteienforschung in Deutschland zu bieten hat.

Christian Nestler

3 Richard S. Katz / William Crotty (Hrsg.), Handbook of Party Politics, London / New Dehli 2006. 